\title{
冷金属过渡电弧增材制造多道搭接工艺研究"
}

\author{
方学伟 1,2 白 浩 ${ }^{2}$ 姚云飞 1,2 张丽娟 ${ }^{1,2}$ 卢秉恒 1,2 \\ (1. 西安交通大学机械制造系统工程国家重点实验室 西安 710049; \\ 2. 国家增材制造创新中心 西安 710075)
}

\begin{abstract}
摘要: 基于冷金属过渡(CMT)电弧增材制造技术, 以 2319 铝合金为堆积材料, 恒定送丝速度与不同焊接速度得到 6 组单层焊 道, 分别采用标准曲线对单层焊道截面轮廓进行拟合, 结果表明抛物线和圆弧曲线的拟合效果均较好。在损失较小模型精度 的情况下简化搭接模型, 基于抛物线曲线建立单层多道斜顶搭接模型, 理论推导最优搭接间距为 0.715 倍单道宽度。为了验 证模型的正确性, 采用不同搭接间距成形单层两道进行试验验证, 并将其与传统的平顶搭接模型进行对比。试验结果表明斜 顶模型更加符合 CMT 电弧增材制造工艺过程。当搭接间距确定后, 研究表明当单层焊道成形高度降低时, 搭接表面不平度 随之减小, 有利于后续多层多道堆积成形。基于优化后的搭接参数, 成形多层多道样件, 成形形貌良好, 三个方向的拉伸力 学性能表现出各向异性。
\end{abstract}

关键词：冷金属过渡；增材制造；搭接模型；多层多道

中图分类号: TG444

\section{Research on Multi-bead Overlapping Process of Wire and Arc Additive Manufacturing Based on Cold Metal Transfer}

\author{
FANG Xuewei $^{1,2}$ BAI Hao $^{2}$ YAO Yunfei ${ }^{1,2}$ ZHANG Lijuan ${ }^{1,2}$ LU Bingheng ${ }^{1,2}$
}

(1. The State Key Laboratory for Manufacturing Systems Engineering, Xi'an Jiaotong University, 710049;

2. National Innovation Institute of Additive Manufacturing, Xi'an 710075)

\begin{abstract}
Multi-bead overlapping process of wire and arc additive manufacturing (WAAM) based on the cold metal transfer (CMT) is studied systematically. By using 2319 aluminum wire as the filling material, experiments are carried out with the constant wire-feed rate and various travel speeds. The cross section's morphology of the obtained single beads are separately fitted by the three selected models, including the parabola model, arc model and cosine model. The experimental results showed that both the parabola and arc functions could accurately represent the bead profile. Based on parabola model, the single-layer multi-pass overlapping model is then established. The theoretical optimal overlapping width is calculated to be 0.715 times that of the layer width. To verify the accuracy of this model, single-layer two-bead samples are produced with different overlapping rates, including the traditional flat-topped overlapping rate 0.6 . The experimental results showed that the model proposed is more in line with the manufacturing process of WAAM based on the CMT. When the overlapping rate is determined, the smaller the single-layer height is, the better would the formed surface profile be, which is propitious for the subsequent multi-layer multi-bead parts fabricating. Based on the optimized overlapping parameters, the multi-layer multi-bead part was fabricated with good surface appearance. And the mechanical properties in three directions are analyzed as well.
\end{abstract}

Key words: CMT(cold metal transfer); additive manufacturing; overlapping model; multi-layer multi-bead

\section{0 前言}

增材制造(Additive manufacturing, AM)将零件的 $\mathrm{CAD}$ 数据切片分层, 使三维零件转变为一系列二维

* 国家重点研发计划资助项目(2018YFB1106302)。20190124 收到初稿, 20190707 收到修改稿
轮廓信息，根据二维轮廓信息逐点、逐面堆积材料 的方法制造实体零件。该技术不需要传统加工的刀 具、夹具及多道加工程序, 是一种自下而上的自由 制造方式 ${ }^{[1]}$ 。特别是金属增材制造已发展成为制造技 术领域新的研究热点，根据原材料的物理形状将金 属增材制造技术分为粉末增材制造和填丝增材制造 两种方式 ${ }^{[2-3]}$ ，基于粉末的增材制造技术常用来成形 
形状精度高, 结构复杂, 成形尺寸较小的零件, 而基 于送丝的电弧增材制造技术以电弧作为热源, 成形的 零件化学成分均匀, 致密度高, 成形尺寸不受成形腔 限制, 设备和原材料成本低, 成形效率高 ${ }^{[4-6]}$, 尤其 适合中等复杂程度大尺寸构件的快速增材制造。

电弧增材制造过程多道搭接工艺首先要解决搭 接间距的设置, 通过多道搭接模型得到理想的搭接 间距, 国内外已有相关研究。搭接模型建立需首先 得到单层焊道截面轮廓的函数表达, 通常采用圆弧、 余弦函数、抛物线进行轮廓的拟合。学者已提出一 种横向搭接平顶模型 ${ }^{[7]}$, 见图 1 所示, 单层焊道截面 轮廓基于抛物线函数, 为了使得成形表面平滑, 理 想搭接间距的条件应使重合区域的面积(曲边三角形 $A E B$ )与波谷区域的面积相等(曲边三角形 $C D E$ )。根 据理想搭接条件求得理想搭接间距 $d=2 / 3 w$ 。

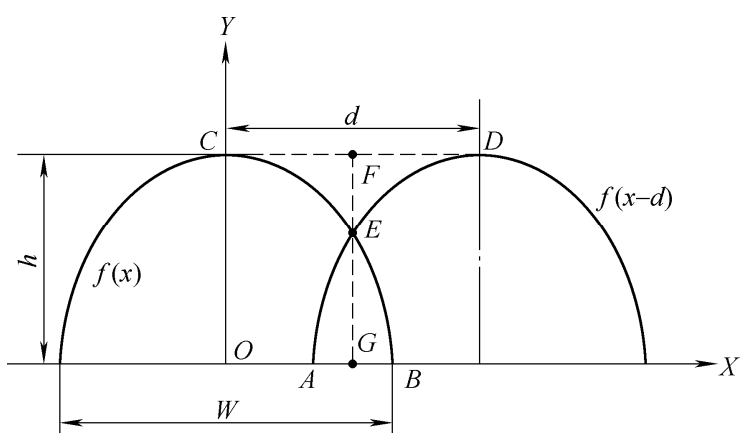

图 1 焊道搭接数学模型

基于熔化极气体保护焊发展而来的冷金属过渡 技术，作为铝合金电弧增材制造的一种高效技术手 段近年来得到了广泛的研究。前文提到虽有学者研 究电弧增材过程的搭接模型, 但对于 CMT 电弧增 材制造中的单层焊道截面轮廓分析和搭接模型相关 的研究较少, 而且采用平顶模型得到的搭接试验件, 并不能实现理想的搭接表面 ${ }^{[8-9]}$, 实际的搭接表面 仍存在一定的波纹度。因此需要建立一种和实际搭 接表面更为接近的数学模型来优化多道的成形工 艺 ${ }^{[10]}$, 本文将采用圆弧、抛物线和余弦函数对单层 焊道截面轮廓进行拟合, 以寻找较优轮廓拟合函数。 基于单道截面拟合函数, 提出一种单层多道斜顶搭 接模型, 并进行试验验证。在此基础上, 开展单层 多道样件和多层多道块件的成形, 同时对其拉伸力 学性能进行分析, 加速 CMT 电弧增材制造技术在 工程技术领域的应用。

\section{1 单层焊道截面轮廓拟合数值模型}

\section{1 三种标准拟合函数}

根据单层焊道横截面轮廓与成形基板所形成的
铺展角 $\theta$ 一般分为两类: 一类 $\theta$ 小于 $90^{\circ}$, 见图 $2 \mathrm{a}$; 另一类 $\theta$ 大于 $90^{\circ}$ ，见图 $2 \mathrm{~b}$ 。采用 $\theta$ 小于 $90^{\circ}$ 的 单层焊道进行横向搭接易出现层间结合空隙, 为了 保证横向搭接的质量, 应选用 $\theta$ 大于 $90^{\circ}$ 的单层焊 道进行搭接工艺的研究。

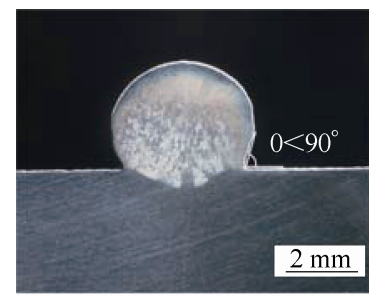

(a) 铺展角 $\theta$ 小于 $90^{\circ}$

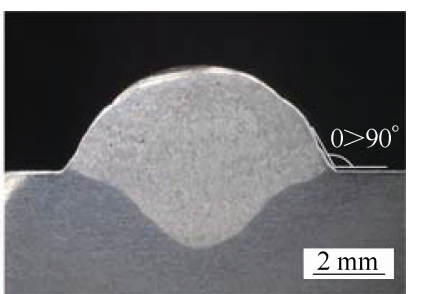

(b) 铺展角 $\theta$ 大于 $90^{\circ}$
图 2 单层焊道截面轮廓

单层焊道截面的几何建模如图 3 所示, 图中 $h$ 代表焊道的高度, $w$ 代表焊道的宽度。分别采用抛 物线、余弦以及圆弧曲线, 对单层焊道截面轮廓进 行拟合, 根据 $B$ 点 $(w / 2,0)$ 和 $C$ 点 $(0, h)$ 坐标可得用 $w$ 和 $h$ 表示的三个函数，如表 1 所示。

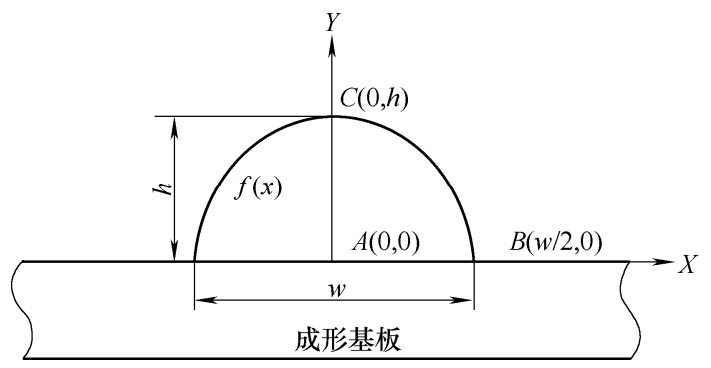

图 3 单道截面轮廓数学模型

表 1 三种曲线函数表达式

\begin{tabular}{cccc}
\hline 类型 & 函数表达式 & $w$ & $h$ \\
\hline 抛物线 & $y=a x^{2}+c$ & $w=2 \sqrt{-\frac{c}{a}}$ & $h=c$ \\
余弦 & $y=a \cos (b x)$ & $w=\frac{b}{\pi}$ & $h=a$ \\
圆弧 & $y=\sqrt{a^{2}-x^{2}}+b$ & $w=2 \sqrt{a^{2}-b^{2}}$ & $h=a+b$ \\
\hline
\end{tabular}

\section{2 试验方法}

本试验采用的是机器人-CMT 电弧增材制造系 统, 如图 4 所示, KUKA 六自由度机器人作为运动 执行机构, 以 FRONIUS -CMT Advanced 4000 R 焊 接电源作为热源, 以 2219-T87 轧制态铝合金板材为 成形基板，尺寸为 $500 \mathrm{~mm} \times 500 \mathrm{~mm} \times 30 \mathrm{~mm}$ 。焊 丝采用抚顺东工生产的 ER2319 铝合金, 直径为 1.2 $\mathrm{mm}$, 焊丝中铜元素的质量百分数为 $5.8 \% \sim 6.8 \%$ 。 保护气体纯度为 $99.99 \%$ 的氩气, 保护气流量为 22 $\mathrm{L} / \mathrm{min}$, 干伸长 $13 \mathrm{~mm}$, 基板温度为 $60{ }^{\circ} \mathrm{C}$ 。焊接电 源选择 $\mathrm{CMT}+\mathrm{P}$ 模式, 送丝速度(WFS)恒定, 不同焊 接速度 $(T S)$ 的取值如表 2 所示。 


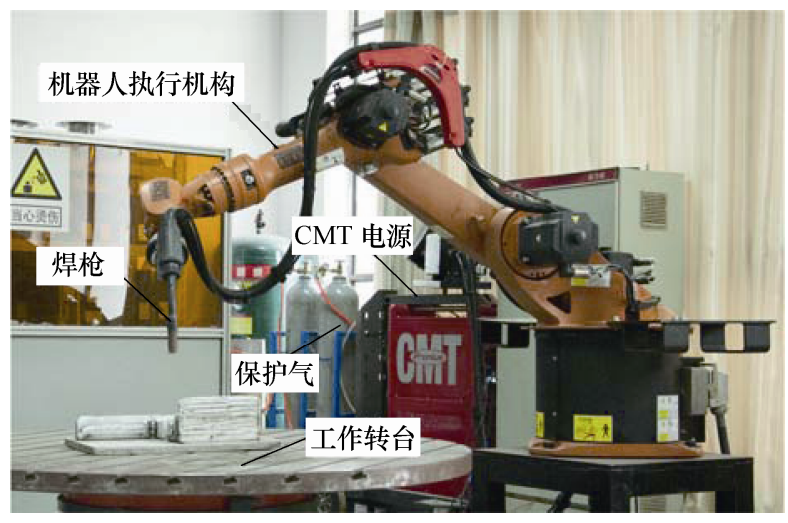

图 4 CMT 电弧增材机器人制造系统

表 2 成形工艺参数

\begin{tabular}{ccccccc}
\hline 试验序号 & 1 & 2 & 3 & 4 & 5 & 6 \\
\hline $\begin{array}{c}\text { 送丝速度/ } \\
\left(\mathrm{m} \cdot \mathrm{min}^{-1}\right)\end{array}$ & 7 & 7 & 7 & 7 & 7 & 7 \\
$\begin{array}{c}\text { 焊接速度/ } \\
\left(\mathrm{m} \cdot \mathrm{min}^{-1}\right)\end{array}$ & 0.48 & 0.56 & 0.64 & 0.72 & 0.80 & 0.88 \\
\hline
\end{tabular}

\section{3 单层焊道截面轮廓拟合结果分析}

对单层焊道试样使用线切割沿垂直于扫描方 向切开获得的单层焊道横截面, 经打磨、抛光、 腐蚀后, 采用体视显微镜观测其宏观形貌, 如图 5 所示。

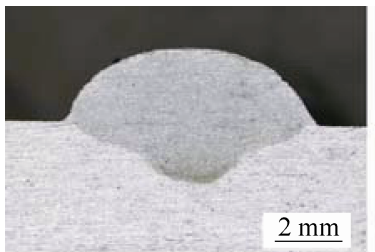

(a) 参数 1

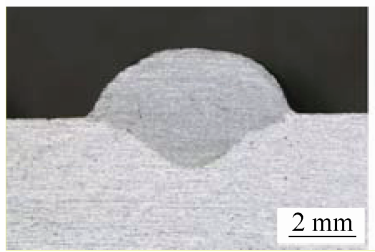

(c) 参数 3

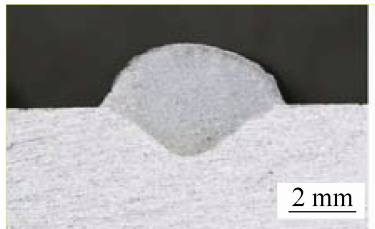

(e) 参数 5

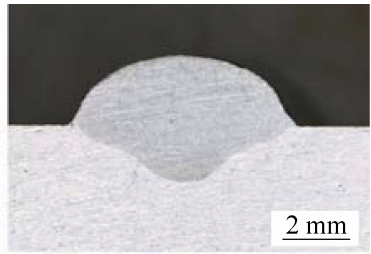

(b) 参数 2

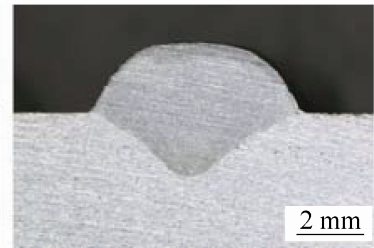

(d) 参数 4

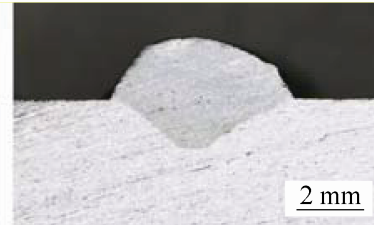

(f) 参数 6
图 5 单层焊道横截面宏观形貌

采用图像测量软件获取单层焊道横截面轮廓 信息, 如图 6 所示, 建立坐标系, 对整个焊道截 面轮廓均匀选取 23 个点, 获得其坐标数据, 通过 Matlab 分别与所选的标准曲线进行拟合, 计算结 果见图 7。

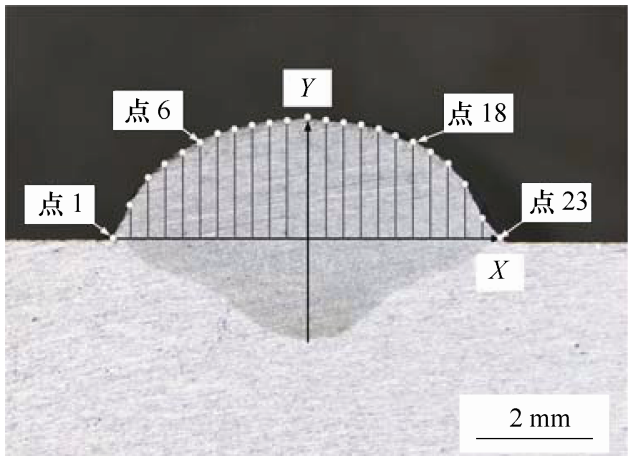

图 6 单层焊道横截面测试点选取

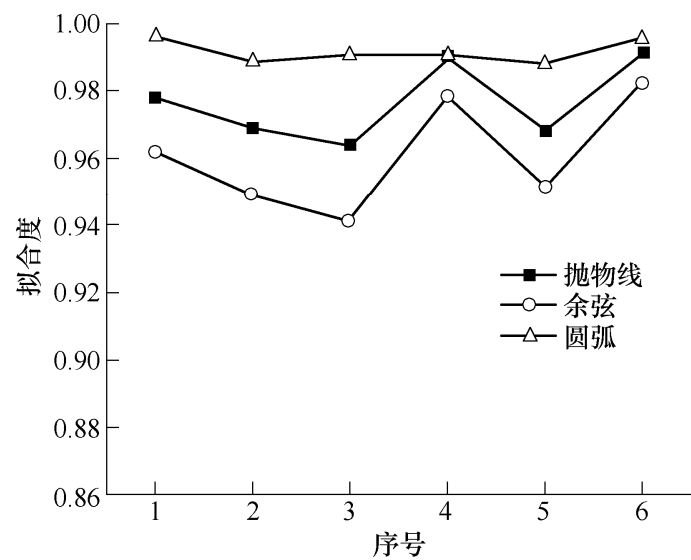

图 7 不同函数拟合度变化规律

由图 7 可得, 三种标准曲线总体来说均表现出 较高的拟合度，拟合度的变化范围在 $0.940 \sim 0.995$ 之间, 余弦函数的拟合度最差, 抛物线和圆弧曲线 的拟合度一直维持在较高的水平，其中圆弧和抛物 线拟合度的平均值分别为 0.991 和 0.977 , 均能较好 地拟合截面轮廓。

圆弧曲线虽然为最优的拟合曲线，为了减少搭 接模型不必要的复杂度, 在损失较小的模型精度的 情况下, 采用较简单的抛物线曲线进行单层多道搭 接模型的建立。

\section{2 单层多道斜顶搭接模型}

在获取单道形貌较为精准的信息之后, 控制相 邻焊道之间的搭接间距 $d$ 是影响单层多道成形的关 键因素。 $d$ 对表面成形精度起着极为重要的作用, 不同的 $d$ 会导致表面成形精度产生较大差别。当 $d$ 大于等于单道宽度的时候，在两道相邻的焊缝之间 没有搭接材料, 此时表现为未搭接, 如图 8a 所示; 随着 $d$ 的减小, 相邻焊缝之间出现波谷部分, 此时 搭接情况表现为欠搭接, 如图 $8 \mathrm{~b}$ 所示; 当两条焊道 重叠区域的面积等于波谷区域的面积时，搭接表面 会接近于理想平面, 此时称为理想搭接间距。此时 为理想搭接, 如图 $8 \mathrm{c}$ 所示; 当 $d$ 继续减小, 重叠区 
域面积的增加会形成凸起的波峰, 破坏搭接处的表 面平整度，如图 $8 \mathrm{~d}$ 所示。

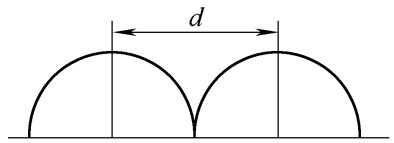

(a) 未搭接

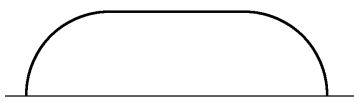

(c) 理想搭接

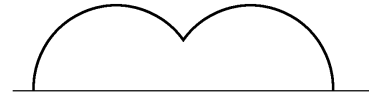

(b) 搭接不足

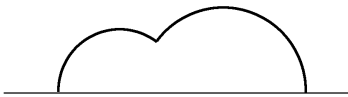

(d) 过度搭接
图 8 四种搭接情况

\section{1 模型建立}

本文所建立的模型为单层多道斜顶搭接模型, 与传统的平顶搭接模型理想搭接的条件一致, 其改 进的地方在于, 当两条焊道横向搭接时, 重合区域 的液态金属在波谷区域的填充不是均匀的, 其凝固 时自由界面并不能形成理想的平面, 因此将其假定 为抛物线曲面, 取横截面进行分析, 具体数学模型 如图 9 所示。基于此, 当理想搭接间距为 $d$ 时, 定 义 $f_{2}(x)$ 为过曲线 $F D$ 的抛物线函数, $A$ 点为第二条 焊道起始点, $F$ 点与 $A$ 点的 $X$ 坐标相等, $A$ 点坐标 根据几何关系为 $(d-w / 2,0), D$ 点为第二条焊道的对 称中点, $D$ 点坐标为 $(d, h)$, 根据理想的搭接条件, 可得 $S_{A E B}=S_{F D E}$ 。

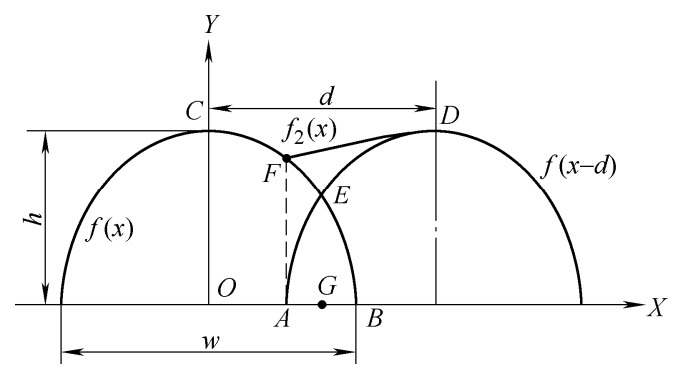

图 9 单层多道斜顶搭接数学模型

曲边三角形 $S_{A E B}$ 为

$$
\begin{gathered}
S_{A E B}=2 S_{E B G}= \\
\int_{d / 2}^{w / 2} f(x) \mathrm{d} x=\frac{h w}{3}+\frac{h d^{3}}{3 w^{2}}-h d
\end{gathered}
$$

曲边三角形 $S_{\mathrm{FDE}}$ 为

$$
S_{F D E}=S_{A F D}-S_{A E F}
$$

其中曲边三角形 $S_{\mathrm{AEF}}$ 为

$$
\begin{gathered}
S_{A E F}=S_{A F B}-S_{A B E}= \\
\int_{d-w / 2}^{w / 2} f(x) \mathrm{d} x-\frac{h w}{3}-\frac{h d^{3}}{3 w^{2}}+h d= \\
\frac{h w}{2}+\frac{h(2 d-w)^{3}-2 h d^{3}}{6 w^{2}}
\end{gathered}
$$

$f_{2}(x)$ 为抛物线曲线，通过 $F\left(d-w / 2,4 h d(w-d) / w^{2}\right)$ 和 $D(d, h)$ 两点。为了便于计算 $S_{\mathrm{AFD}}$ 的面积，将 $f_{2}(x)$ 和 $f(x-d)$ 沿 $X$ 轴向负方向平移 $d$, 示意图见图 10 。

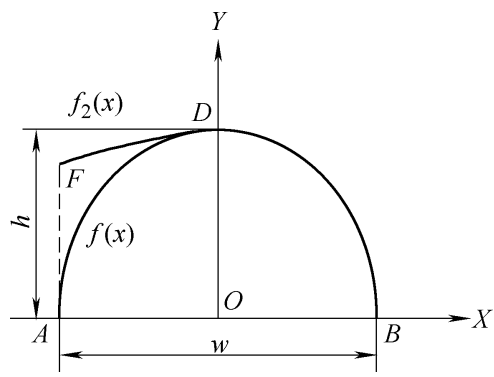

图 10 平移之后的第二条焊道坐标

平移后 $f_{2}(x)$ 通过 $F$ 点 $\left(-w / 2,4 h d(w-d) / w^{2}\right)$ 和 $D(0, h)$ 两点的抛物线, 即可求出平移后 $f_{2}(\mathrm{x})$ 的表达 式为

$$
f_{2}(x)=-\frac{4 h}{w^{4}}(2 d-w)^{2} \times x^{2}+h
$$

即有

$$
\begin{gathered}
S_{A F D}=\int_{-w / 2}^{0}\left[f_{2}(x)-f(x)\right] \mathrm{d} x= \\
\frac{h w}{6}-\frac{h(2 d-w)^{2}}{6 w}
\end{gathered}
$$

此时可得 $S_{F D E}$ 的面积为

$$
S_{F D E}=S_{A F D}-S_{A E F}=h\left[\frac{d^{3}}{3 w^{2}}-\frac{w}{3}-\frac{d(2 d-w)^{2}}{3 w^{2}}\right]
$$

定义曲边三角形 $S_{F D E}$ 和 $S_{A E B}$ 的差值为 $g(x)$, 则

$$
\begin{gathered}
g(x)=S_{F D E}-S_{A E B}= \\
-\frac{4}{3 w^{2}} d^{3}+\frac{4}{3 w} d^{2}+\frac{2}{3} d-\frac{2}{3} w
\end{gathered}
$$

当理想的搭接时, 令 $g(x)=0$, 利用 Matlab 求解 此方程, 只有两个实数根即

$$
d_{1}=w \quad d_{2}=0.715 w
$$

$d_{1}$ 等于 $w$ 显然是不合理，因此斜顶模型理想搭 接间距为 $0.715 w$ 。

\section{2 试验验证}

为了验证单层多道斜顶搭接模型, 在 $\mathrm{CMT}+\mathrm{P}$ 模式下取焊接速度 $0.5 \mathrm{~m} / \mathrm{min}$, 送丝速度 $7 \mathrm{~m} / \mathrm{min}$, 保护气流量控制为 $22 \mathrm{~L} / \mathrm{min}$, 干伸长量为 $13 \mathrm{~mm}$, 搭接间距设置如表 3 所示, 进行四组单层两道搭接 试验。

\section{表 3 搭接间距设置}

\begin{tabular}{ccccc}
\hline 试验编号 & 1 & 2 & 3 & 4 \\
\hline$d$ & $0.6 w$ & $0.667 w$ & $0.715 w$ & $0.8 w$ \\
\hline
\end{tabular}


用线切割将所得试样沿垂直于扫描方向切开, 经打磨、抛光、腐蚀后, 采用体视显微镜拍摄单层 两道搭接截面宏观形貌, 如图 9 所示。图中虚线和 基板平面平行, 当搭接间距为 $0.6 \mathrm{w}$ 时, 如图 $11 \mathrm{a}$ 所 示, 搭接截面轮廓出现了两个波峰, 此时搭接重叠 部分的面积大于波谷区域, 波谷区域被完全填充, 但是使得第二条焊道的高度增加, 两个波峰的垂直 距离为 $0.73 \mathrm{~mm}$, 表现为过搭接状态。当搭接间距 为 $0.667 w$ 时, 即平顶模型所计算的理想搭接间距, 截面形貌出现了两个波峰和一个波谷, 如图 $11 \mathrm{~b}$ 所 示, 波谷区域不会形成一个理想的平面, 最高波峰 与波谷的垂直距离为 $0.84 \mathrm{~mm}$, 也表现为过搭接的 状态。这是由于搭接时熔体自身表面张力的作用下, 重合区域凝固时自由界面会收缩为曲面。当搭接间 距为 $0.715 w$ 时, 即将重合区域凝固时自由界面假定 为抛物线曲面, 所建立的单层多道斜顶搭接模型推 导的理想搭接间距, 截面形貌出现了两个波峰和一 个波谷, 如图 11c 所示, 两个波峰基本在同一水平, 波峰与波谷垂直距离为 $0.51 \mathrm{~mm}$, 此时搭接间距设 置合理。当搭接间距为 $0.8 w$ 时, 截面形貌出现了明 显的波谷, 两个波峰也基本在同一水平, 波峰与波 谷垂直距离为 $0.88 \mathrm{~mm}$, 此时搭接状态表现为欠搭 接, 如图 11d 所示。

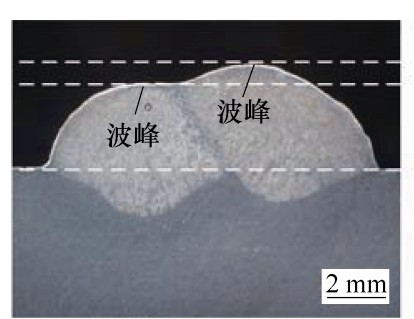

(a) $d=0.6 w$

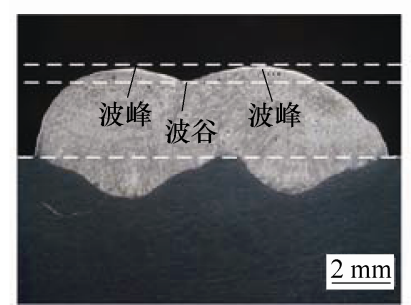

(c) $d=0.715 w$
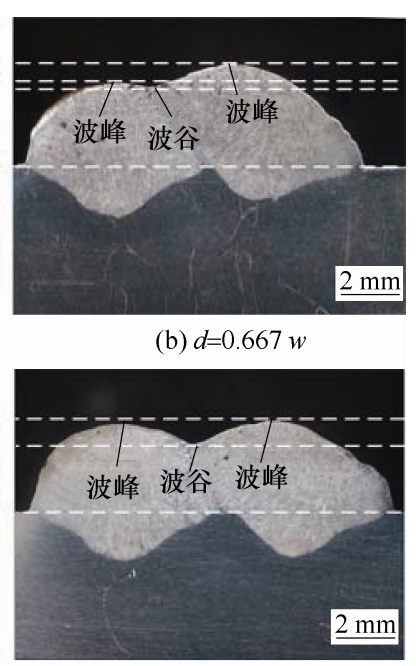

(d) $d=0.8 \mathrm{w}$ (b) $d=0.667 \mathrm{w}$

图 11 不同搭接间距下单层两道搭接截面形貌

综上所述, 当搭接间距为 $0.715 w$ 时, 搭接质量 最理想, 证明了将重合区域凝固时自由界面假定为 抛物线曲面, 更加接近真实情况, 所建立的斜顶搭 接模型比平顶搭接模型更加适用于 CMT 电弧增材 制造工艺。

\section{3 搭接表面不平度变化规律研究}

当搭接间距确定之后, 研究表明单层焊道成形
高度对与搭接表面波峰与波谷的垂直距离有重大影 响。为了寻找最优搭接工艺, 增加两组试验与上述 验证试验最优结果进行对比, 将单层多道斜顶搭接 模型试验的焊接速度 $(T S$ )数值按照 1.4 倍等比例变 为 $0.7 \mathrm{~m} / \mathrm{min}$ 和 $0.357 \mathrm{~m} / \mathrm{min}$, 其他工艺参数不变, 搭接间距均为 $0.715 w$, 试验结果如图 12 所示。

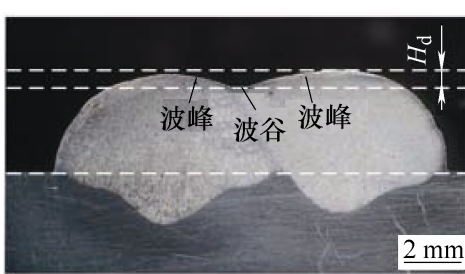

(a) $T S=0.357 \mathrm{~m} / \mathrm{min}$

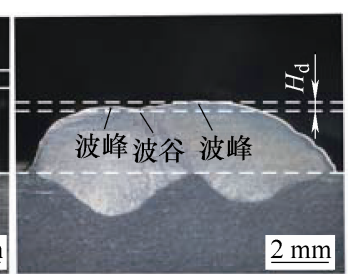

(b) $T S=0.7 \mathrm{~m} / \mathrm{min}$
图 12 不同焊接速度下单层两道搭接截面形貌

为了定量的描述搭接表面的成形质量, 定义搭 接表面波峰与波谷之间在垂直方向上的距离为表 面不平度 $H_{d}$, 如图 12 所示, 显然 $H_{d}$ 值越大则搭 接表面的成形精度越差。分别计算三种不同焊接速 度下的单层两道搭接截面 $H_{d}$, 横坐标为单层焊道 的高度, 结果如图 13 所示, 并对数据进行线性拟 合, $R^{2}$ 为 0.96 , 因此当搭接间距确定后, $H_{d}$ 与单 层焊道成形高度成线性相关。当单道成形高度降低 时, $H_{d}$ 也随之减小，搭接表面越平整。因此当搭 接间距选定后, 可以选择成形高度较小的单层焊 道, 虽然降低了成形效率, 但可以获得较为平整的 搭接表面, 有利于后续多层多道堆积成形, 且提高 成形的精度。

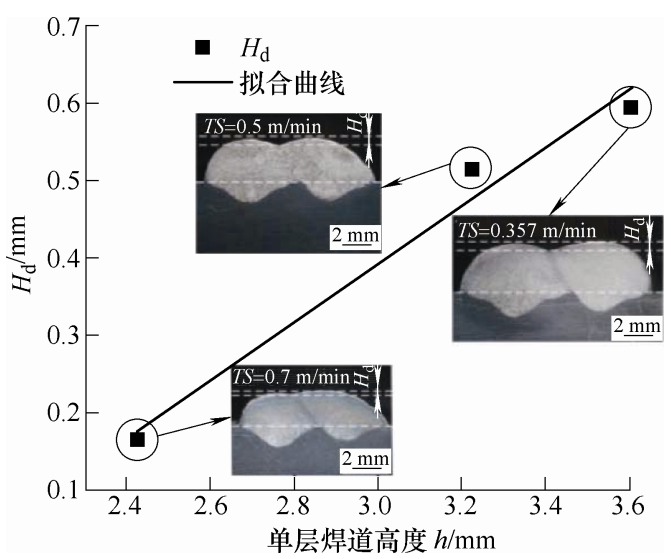

图 13 不同单层焊道成形高度下 $H_{d}$ 的变化规律

根据上述结论, 进行单层多道的成形, 工艺参 数选择焊接速度为 $0.7 \mathrm{~m} / \mathrm{min}$, 送丝速度为 $7 \mathrm{~m} / \mathrm{min}$, 搭接间距为 $0.715 w$ 时, 搭接 29 道, 其表面形貌及 沿垂直于扫描方向截取的横截面如图 14 所示, 从横 截面可以看出单层多道搭接表面非常平整, 几乎没 有较大的波峰和波谷, 其中间位置的平均表面不平 度仅为 $0.27 \mathrm{~mm}$, 成形精度较高。 


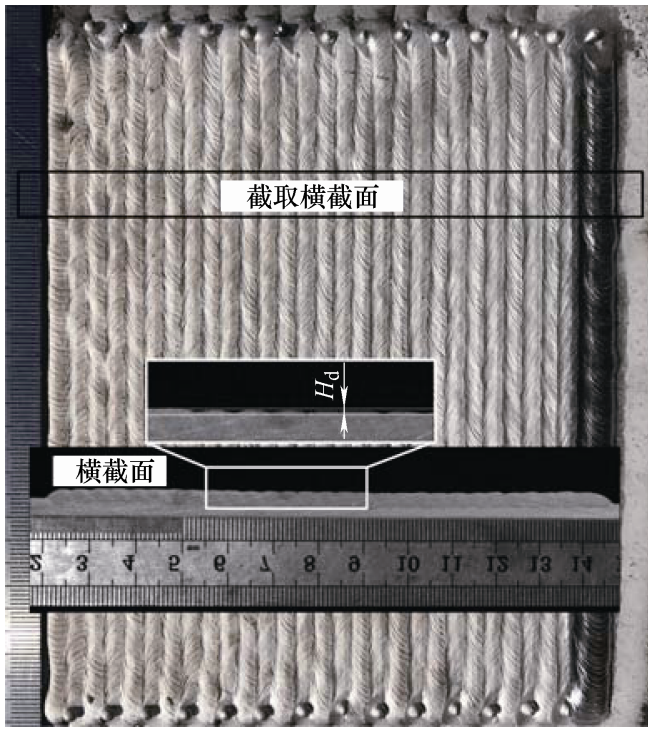

图 14 单层多道成形表面及横截面

\section{3 多层多道块体成形试验}

基于上述单层多道成形搭接工艺研究, 开展多 层多道成形试验, 采用的工艺参数如下焊接速度为 $0.7 \mathrm{~m} / \mathrm{min}$, 送丝速度为 $7 \mathrm{~m} / \mathrm{min}$, 选择 CMT+P 模式, 搭接间距设定为 $0.715 w$, 成形了如图 15 所示, 直 径 $400 \mathrm{~mm}$, 高 $150 \mathrm{~mm}$, 壁厚 $20 \mathrm{~mm}$ 的圆筒结构, 并对顶部进行了减材加工, 可以看出成形良好, 无 不良搭接缺陷。进一步增加搭接道数, 成形了如图 16 所示的典型法兰盘结构, 法兰盘外径 $380 \mathrm{~mm}$, 内径 $270 \mathrm{~mm}$, 以及带有凸面的法兰, 均可保持较高 的成形精度。
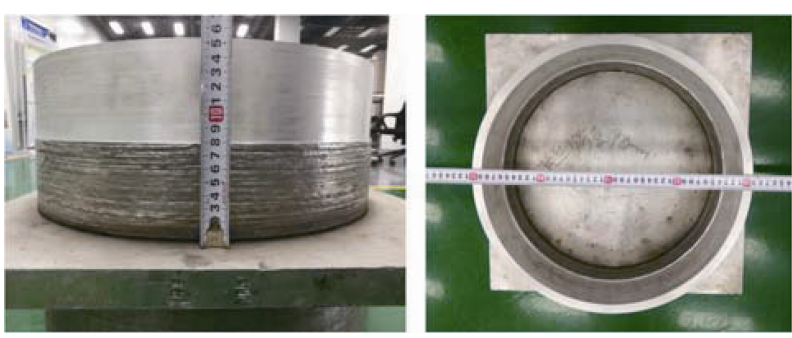

图 15 典型厚壁圆筒件成形
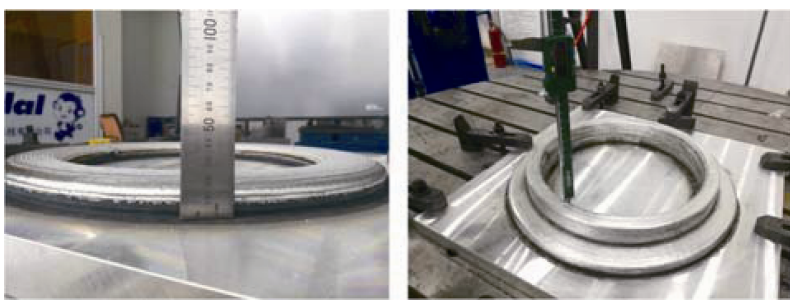

图 16 典型法兰盘结构

为了测试多层多道搭接的力学性能, 采用层间 首尾交替扫描方式进行多层多道块体的成形, 如图 17 所示。工艺参数选择焊接速度为 $0.7 \mathrm{~m} / \mathrm{min}$, 送
丝速度为 $7 \mathrm{~m} / \mathrm{min}$, 搭接间距为 $0.715 \mathrm{w}$, 所成形的 块体的尺寸为 $220 \mathrm{~mm} \times 100 \mathrm{~mm} \times 70 \mathrm{~mm}$, 如图 18 所示, 块体外观良好, 无明显外观缺陷。

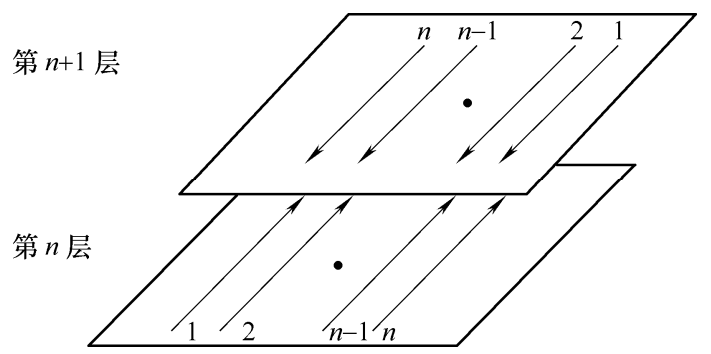

图 17 层间首尾交替扫描方式

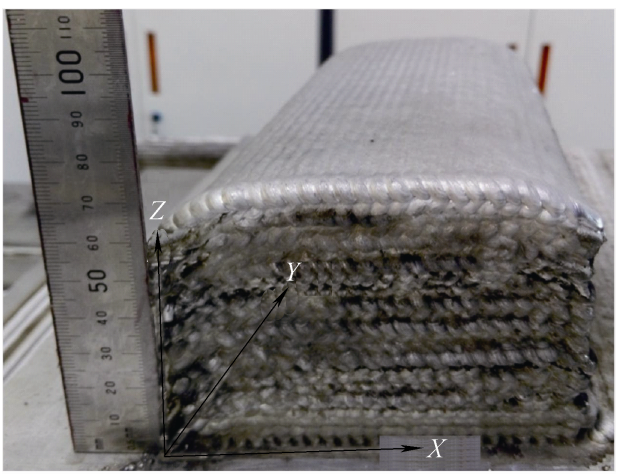

图 18 多层多道块体

根据 GB/T 228.1-2010 标准, 分别沿搭接方向 $X$ 、扫描方向 $Y$ 及堆积方向 $Z$ 切割拉伸试样, 拉伸 试样选取见图 19 所示, 采用金相砂纸去除拉伸试样 表面线切割痕迹，每个方向试样均选择 3 个。

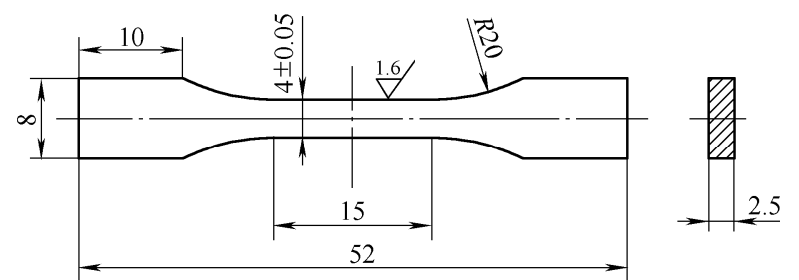

图 19 拉伸试样尺寸

采用 INSTRON1195 材料试验机进行测试，室 温条件, 拉伸速度为 $1 \mathrm{~mm} / \mathrm{min}$, 拉伸结果如图 19 所示。试验结果显示多层多道块体搭接方向 $X$ 、扫 描方向 $Y$ 及堆积方向 $Z$ 的抗拉强度的平均值分别为 $256.67 \mathrm{MPa} 、 270.67 \mathrm{MPa}$ 和 $246.67 \mathrm{MPa}$ 。扫描方向 $Y$ 的抗拉强度大于搭接方向 $X$ 大于垂直高度方向 $Z$, 各向有较为明显的差异。三个方向的断后伸长率同 样遵循相同的规律，搭接方向 $X$ 、扫描方向 $Y$ 及堆 积方向 $Z$ 的断后伸长率的平均值分别为 $13.33 \%$ 、 $21.33 \%$ 和 $12.33 \%$ 。分析其原因主要是沿着 $Y$ 扫描方 向的拉伸试件在每层成形轨迹上填充金属大多是连 续成形, 并且与拉伸受力方向相同, 而在方向 $X$ 和 方向 $Z$ 则存在着众多焊道搭接, 在道间搭接区域, 
气孔容易聚集, 微观组织相对不均匀, 为拉伸性能 薄弱区, 从而导致力学性能相比 $Y$ 方向均有下降, 表现出各向异性。

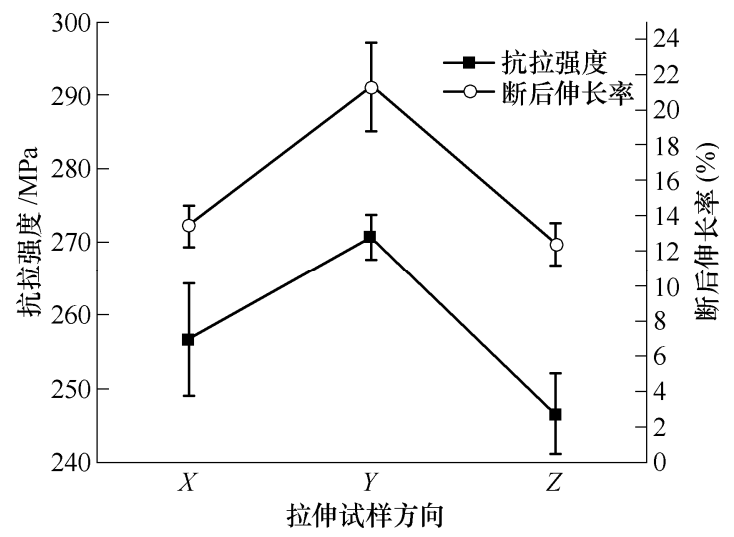

图 20 拉伸试件拉伸强度及断后伸长率

\section{4 结论}

(1) 圆弧和抛物线对单层焊道截面轮廓的拟合 效果均较好。基于抛物线函数提出一种单层多道斜 顶搭接模型，计算最优搭接间距为 $0.715 w$ 。

(2) 试验验证了提出的单层多道斜顶搭接模型 更加适用于 CMT 电弧增材制造工艺。当搭接间距 确定后, 表面不平度与单层焊道高度基本呈线性相 关。选择成形高度较小的单层焊道, 可以提高搭接 表面成形的精度。

（3）基于单层多道斜顶搭接模型，进行多层多 道块体成形, 成形形貌良好, 扫描方向、搭接方向 和堆积方向的拉伸力学性能表现为各向异性。

\section{参 考 文 献}

[1] 李涤尘, 贺健康, 田小永, 等. 增材制造: 实现宏微结 构一体化制造[J]. 机械工程学报, 2013, 49(6): 129-135. LI Dichen, HE Jiankan , TIAN Xiaoyong , et al. Additive manufacturing : Integrated fabrication of macro /microstructures [J]. Journal of Mechanical Engineering, 2013, 49(6): 129-135.

[2] SUI H M, BI G, FOLKES J, et al. Deposition of $\mathrm{Ti}-6 \mathrm{Al}-4 \mathrm{~V}$ using a high power diode laser and wire, Part I: Investigation on the process characteristics[J]. Surface
\& Coatings Technology, 2008, 202(16): 3933-3939.

[3] BRANDL E, MICHAILOV V, VIEHWEGER B, et al. Deposition of Ti-6Al-4V using laser and wire, part I: Microstructural properties of single beads[J]. Surface \& Coatings Technology, 2011, 206(6): 1120-1129.

[4] FANG Xuewei, ZHANG Lijuan, LI Hui, et al. Microstructure evolution and mechanical behavior of 2219 aluminum alloys additively fabricated by the cold metal transfer process[J]. Materials, 2018, 11(5): 812.

[5] DONOGHUE J, ANTONYSAMY A A, MARTINA F, et al. The effectiveness of combining rolling deformation with wire-arc additive manufacture on $\beta$-grain refinement and texture modification in $\mathrm{Ti}-6 \mathrm{Al}-4 \mathrm{~V}[\mathrm{~J}]$. Materials Characterization, 2016, 114: 103-114.

[6] SHEN C, PAN Z, CUIURI D, et al. Fabrication of Fe-FeAl functionally graded material using the wire-arc additive manufacturing process[J]. Metallurgical \& Materials Transactions B, 2016, 47(1): 763-772.

[7] XIONG Jun, ZHANG Guangjun, GAO Hongming, et al. Modeling of bead section profile and overlapping beads with experimental validation for robotic GMAW-based rapid manufacturing $[\mathrm{J}]$. Robotics and Computer \& Integrated Manufacturing, 2013，29(2): 417-423.

[8] SURYAKUMAR S , KARUNAKARAN K , BERNARD $A$, et al. Weld bead modeling and process optimization in hybrid layered manufacturing[J]. Computer-Aided Design, 2011, 43(4): 331-344.

[9] CAO Y, ZHU S, LIANG X, et al. Overlapping model of beads and curve fitting of bead section for rapid manufacturing by robotic MAG welding process[J]. Robotics \& Computer Integrated Manufacturing, 2011, 27(3): 641-645.

[10] DING D, PAN Z, CUIURI D, et al. A multi-bead overlapping model for robotic wire and arc additive manufacturing (WAAM) $[\mathrm{J}]$. Robotics \& Computer Integrated Manufacturing, 2015，31(C): 101-110.

作者简介: 方学伟, 男, 1989 年出生, 博士研究生。主要研究方向为金 属增材制造。

E-mail: fangxuewei0801@163.com

卢秉恒(通信作者), 男, 1945 年出生, 博士, 教授, 中国工程院院士, 博士研究生导师。主要研究方向为金属增材制造、微纳制造、生物制造、 高速切削机床。

E-mail: bhlu@mail.xjtu.edu.cn 